\title{
EOM-NPOSESs: Emergency Ontology Model Based on Network Public Opinion Spread Elements
}

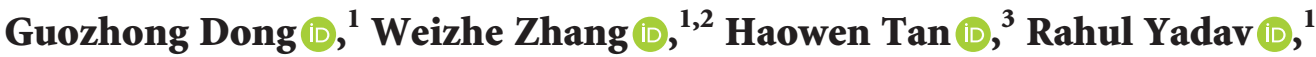 \\ and Shuaishuai Tan $\mathbb{D}^{1}$ \\ ${ }^{1}$ The Cyberspace Security Research Center, Peng Cheng Laboratory, Shenzhen 518000, China \\ ${ }^{2}$ School of Computer Science and Technology, Harbin Institute of Technology, Harbin 150001, China \\ ${ }^{3}$ Department of Computer Engineering, Chosun University, Gwangju 61452, Republic of Korea \\ Correspondence should be addressed to Weizhe Zhang; weizhe.zhang@pcl.ac.cn
}

Received 17 March 2021; Revised 14 April 2021; Accepted 24 May 2021; Published 12 June 2021

Academic Editor: Liguo Zhang

Copyright (c) 2021 Guozhong Dong et al. This is an open access article distributed under the Creative Commons Attribution License, which permits unrestricted use, distribution, and reproduction in any medium, provided the original work is properly cited.

\begin{abstract}
The construction of an emergency ontology model plays an important role in emergency management, which is an important basis for emergency public opinion management and decision-making. Integration of network public opinion spread elements into the emergency ontology model is crucial for realizing knowledge sharing in the field of emergency and public opinion responses. In this study, we crawl a large amount of emergency data from different data sources and construct an emergency dataset. Based on this dataset, we analyze the public opinion elements of emergencies and propose an emergency ontology model based on network public opinion spread elements (EOM-NPOSESs). Thereafter, we consider the coronavirus disease (COVID-19) emergency as an example to construct the EOM-NPOSESs. Finally, we design some strategies to realize rule reasoning and present the COVID-19 emergency application based on the constructed EOM-NPOSESs and the geographic information system platform. The results demonstrate that EOM-NPOSESs can not only describe the semantic relationship between emergencies and emergency elements but also perform semantic logical reasoning on different emergencies.
\end{abstract}

\section{Introduction}

With the rapid development of the social economy and due to socialist modernization, various social emergencies continue to occur and develop. Emergencies have had a tremendous impact on people's lives and are a threat to the normal order of public safety. Emergencies occur suddenly and can lead to a serious social harm. They can be divided into four categories: natural disasters, accident disasters, public health incidents, and social security incidents. Therefore, the public is often concerned and discusses emergency situations, which becomes the focus of public opinion. We are currently in a period of prominent contradictions and fragility of social structures. Facing an increasing number of emergencies, leading cadres at different levels have led to the accumulation of valuable practical experience. Emergencies are caused by several factors, and as such, decision makers must consider all these factors. Whether the government releases information pertaining to emergencies in a timely manner has become a critical factor for calming emergencies.

Research related to emergencies involves public opinion [1-3], network security [4-6], and data mining analysis [7-12]. Ontology models have been widely used in the fields of knowledge engineering and natural language processing. Ali et al. [12] proposed a fuzzy ontology to describe semantic knowledge and its relationships in the transportation domain. Fuzzy ontology-based semantic knowledge can improve deep learning techniques that extract more accurate aspects or features from the transportation text. Modeling domain knowledge using related technologies has become a research focus. To handle various emergencies effectively and reduce the impact of an emergency, it is necessary to perform good ontology modeling, ontology representation, and ontology reasoning for emergencies. Traditional emergency ontology models [13-20] are based on the static emergency concept, which cannot fully describe the 
semantic relationship between emergencies and network public opinion spread elements. These emergency ontology models have significant shortcomings in knowledge fusion, knowledge reasoning, and knowledge questions and answers. Our previous research results [21, 22] demonstrate that the spread and diffusion of online public opinion in similar emergencies have similar burst patterns and key users. Integrating network public opinion spread elements into the emergency ontology model is of great significance in realizing knowledge sharing in the field of emergency and public opinion responses to emergencies.

In this study, emergency data crawled by web crawlers were used to construct an emergency dataset. We analyzed the public opinion elements of emergencies and proposed an emergency ontology model based on network public opinion spread elements (EOM-NPOSESs). Protégé was used to complete the modeling of the emergency ontology model. Coronavirus disease (COVID-19) emergencies were used as examples of emergency ontology models for experimental verification. The main contributions of this study are as follows:

(1) Network public opinion spread elements were integrated to define and describe the emergency ontology model. An EOM-NPOSESs was proposed.

(2) An emergency class and emergency class element construction method was proposed. Protégé was utilized to complete the emergency ontology modeling of COVID-19.

(3) Reasoning rules for COVID-19 emergencies were set based on EOM-NPOSESs. An emergency response prototype system with reasoning rules was developed to verify the applicability of the EOM-NPOSESs.

\section{Related Works}

According to their domain, emergency ontology models can be divided into two categories: general and specific. Research on general emergency ontology models is not limited to a specific type of emergency, making it more widely applicable. Specific domain emergency ontology models can be divided into different categories according to their domains and are often applicable only in those domains. Emergency ontology models can also be divided into single- and multilevel emergency ontology models.

In the domain of single-level semantic models and application research, Bitencourt et al. [13] presented an innovative ontological model for response protocols to fire emergencies in buildings. The conceptual model can be reused using other ontologies and systems related to the fire emergency domain. Several studies have adopted hierarchical methods to model emergencies using multilevel semantic models and applications. Wang et al. [14] proposed the emergency case ontology model comprising two parts: the upper ontology eABC and the application layer ontology. The former was obtained by extending the ABC ontology, whereas the latter from four aspects. Zhu and Liu [15] proposed an emergent event ontology model, which can be divided into three levels: upper-level event type, lower-level event type, and event instance. The model can describe the time, location, object, and relationship between event types. $\mathrm{Ni}$ et al. [16] constructed an emergency knowledge model based on the emergency response plan system in China. The model is divided into three levels: top-level, domain, and application ontologies. Knowledge sharing was achieved by constructing an emergency knowledge system. The focus of the abovementioned emergency studies was to construct a semantic model for emergencies. Liu et al. [17] proposed the concept of the event ontology pattern and its development method, which captures formally reoccurring models and reuses existing emergency ontology vocabulary. Tan et al. [18] proposed a generic ontology pattern for an emergency system model. A set of reasoning rules for emergency evolution, emergency solutions, and emergency resource utilization reasoning was proposed to conduct emergency knowledge reasoning based on the emergency ontology pattern. van Hage et al. [19] presented a simple event model to model events in various domains and provided examples from two use cases: historical emergencies and emergencies in the maritime safety and security domains. Gaur et al. [20] introduced empathy ontology to conceptualize core concepts concerning emergency management and planning of hazard crises.

Research on emergency ontology models has yielded good results, but some problems remain.

It is difficult to find the logical relationship and internal connection of online public opinion spread elements in different emergencies.

Further, although the existing emergency ontology models focus on constructing semantic models, they do not provide sufficient verification of the availability of the model and exploration of its applications, such as semantic reasoning, visual analysis, and semantic association in specific fields.

\section{Emergency Ontology Model Based on Network Public Opinion Spread Elements}

\subsection{Related Definitions and Formal Descriptions}

Definition 1. Emergencies occur suddenly and cause serious social harm, and emergency response measures for natural disasters, accident disasters, public health incidents, and social security incidents must be taken. We use $e$ to represent an emergency; $e$ is composed of six elements that can be represented by a six-tuple as follows:

$$
e=\langle T, P, O, A, \mathrm{EA}, L\rangle
$$

where $T$ denotes the time element, which represents the occurrence time of the emergency; $P$ is the place/location element, which represents the location of the emergency; $O$ is the object element, which represents the objects participating in the emergency, including key users and spread platform of online public opinion; $A$ denotes the action element, which represents the action word that caused the emergency; EA denotes the emotional attitude element, which represents the emotional attitude of netizens 
(positive, neutral, and negative); and $L$ denotes the level element, including the impact level of the emergency and the level of network public opinion.

Definition 2. Emergency set includes a collection of emergencies with common or similar emergency elements.

$$
E S=\left\{E_{1}, E_{2}, E_{3}, \ldots, E_{i}, \ldots\right\},
$$

where $E_{i}=\left\{e_{1}, e_{2}, \ldots, e_{i}, \ldots\right\}, i \in(T, P, O, A, \mathrm{EA}, L)$, and $E_{i}$ represents a collection of emergencies that are specifically identical or similar in terms of emergency elements $i$.

Definition 3. Hierarchy of the emergency set: If there is a hierarchical classification relationship between emergency sets $E S_{1}$ and $E S_{2}, E S_{1}$ is a subset of $E S_{2}$, which is expressed by $R_{i s-\text { sub }}\left(E S_{1}, E S_{2}\right)$. Thus, $E S_{1}$ is referred to as the lower emergency of $E S_{2}$, whereas $E S_{2}$ is referred to as the upper emergency of $E S_{1}$. For instance, an infectious disease emergency is a subordinate emergency category of a public health emergency. It can be expressed as $R_{i s-}$ sub (infectious disease emergency, public health emergency).

Definition 4. Nonhierarchy of emergency: (1) Composition relationship: If the emergency $e_{1}$ comprises the emergency class $e_{2}$, the two emergencies can be defined as having a composition relationship, which can be expressed as $R_{\text {composed }}$. (2) Causal relationship: If the occurrence of an emergency $e_{1}$ leads to an emergency $e_{2}$, the two emergencies can be defined as having a causal relationship, which can be expressed as $R_{\text {causality }}$. (3) Follow relationship: In a specific time range, if the occurrence of emergency $e_{1}$ follows the occurrence of emergency $e_{2}$, the two emergencies can be defined as having a follow relationship, which can be expressed as $R_{\text {following }}$. (4) Concurrency relationship: Emergencies $e_{1}$ and $e_{2}$ occur simultaneously in a specific time range; thus, the two emergencies can be defined as having a concurrent relationship, which can be expressed as $R_{\text {concurrency }}$.

Definition 5. Emergency ontology is a shared and objectively existing emergency model, defined as a five-tuple: $\mathrm{EO}=\langle\mathrm{UES}, \mathrm{LES}, R$, Rules, Individuals $\rangle$. The upper emergency set (UES) has a hierarchical structure, whereas the lower emergency set (LES) has a nonhierarchical structure. Emergency relationship $(R)$ refers to the relationship set between the emergency sets or emergencies. Rules refer to the inference rules, including the inference rules for emergencies and emergency relationships. Individuals represent the emergency instances. The structure of the EOM-NPOSESs is shown in Figure 1. The upper emergency set is a general classification structure, whereas the lower emergency set is a lattice structure formed by the emergency relationships.

\subsection{Emergency Ontology Model Construction}

3.2.1. Emergency Set Construction. The emergency set in the EOM-NPOSESs has a hierarchical structure formed using emergency classification and coding (National Standard of the People's Republic of China GB/T 35561-2017) (https://www. gb688.cn/bzgk/gb/newGbInfo?hcno=4C037B649CB13F556E 00FA63A56528AB), which is divided into three categories. The partial hierarchical classification relationship between the emergency sets is shown in Figure 2.

Emergencies related to natural disasters include seven subcategories: meteorological disasters, floods and droughts, earthquake disasters, geological disasters, marine disasters, biological disasters, and forest and grassland fires.

Emergencies related to accidents include seven subcategories: wars and conflicts, safety production accidents, public facilities and equipment accidents, transportation accidents, environmental pollution accidents, ecological damage accidents, and tourism accidents.

Emergencies related to public health incidents include five subcategories: infectious epidemic incidents, mass disease transmission incidents, occupational hazard incidents, food safety incidents, and animal epidemics.

Emergencies related to social safety incidents include six subcategories: terrorist attacks, foreign-related emergencies, economic security incidents, social hotspot incidents with significant impact, large-scale mass incidents, and riot incidents.

The third layer comprises 80 subcategories. For example, the infectious disease emergencies include plague epidemics, cholera epidemics, pulmonary anthrax epidemics, infectious atypical pneumonia epidemics, human infections with highly pathogenic avian influenza, other epidemics of infectious diseases managed according to category A, epidemics of category $B$ infectious diseases, epidemics of infectious diseases managed according to category $\mathrm{B}$, new infectious diseases or the introduction of infectious diseases that have not yet been discovered, and outbreaks of infectious diseases that have been eliminated.

In addition to the hierarchical relationship of the emergency set, EOM-NPOSESs also include a nonhierarchical emergency relationship, such as composition, following, concurrency, and causality. As shown in Figure 1, the COVID-19 emergency is used as an example to construct a basic emergency relationship structure. After the construction of the COVID-19 nonhierarchical relationship, it is linked to the emergency set in "Other epidemics of infectious diseases managed by category A."

The hierarchy of the emergency set was constructed using an open-source neural hierarchical multilabel text classification toolkit [23] based on the text description of an emergency. It associates emergencies to the corresponding emergency set according to the classification tags of the emergency. The nonhierarchy of the emergency set was manually labeled for association analysis.

3.2.2. Emergency Element Construction. The time ontology from Web Ontology Language (OWL) official recommendations (https://www.w3.org/TR/2017/REC-owl-time-2017 1019) was used in this study. The ontology specification provides a standard vocabulary to describe the topological relationship of a certain instant or period, as well as the 


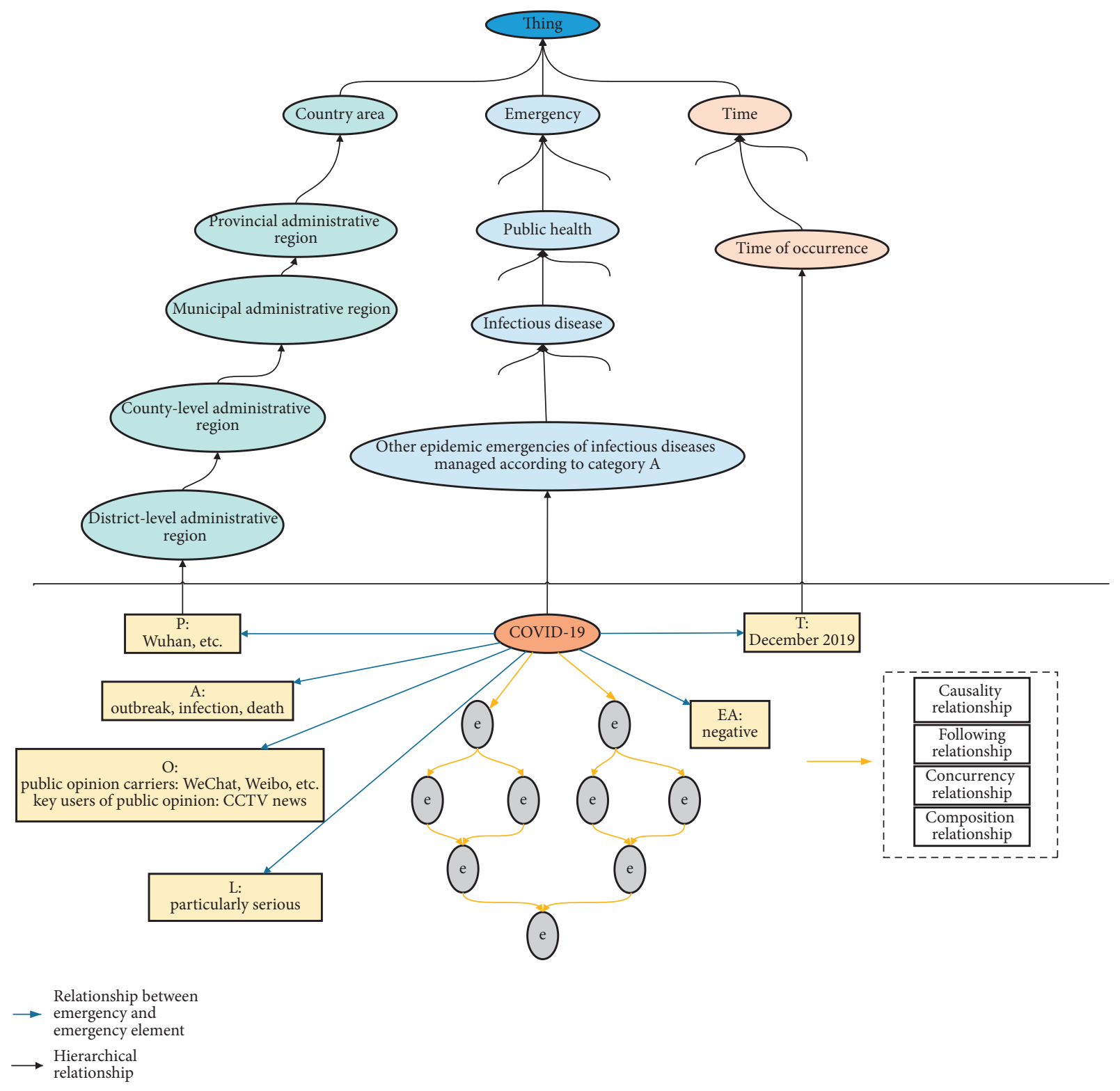

Figure 1: Structure of EOM-NPOSESs.

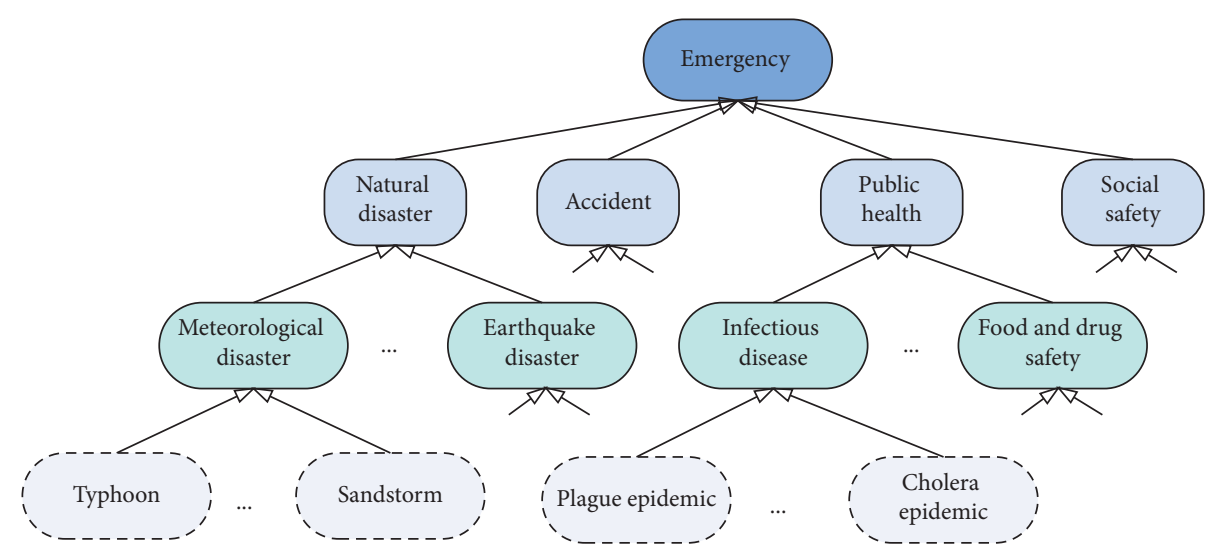

Figure 2: Partial hierarchical classification relationship in an emergency set. 
duration and other information. The ontology also contains additional information related to date and time. In this study, the time element of emergency was taken as an instance of the corresponding time ontology model, and this element can be represented using the traditional calendar or other calendars as well, such as Unix-time or geological time.

According to the place name/address hierarchy rules and coding method in the national standard of the People's Republic of China-Digital City Geographic Information Public Platform Place Name/Address Coding Rules (GB/T 237052009) (http://www.gb688.cn/bzgk/gb/newGbInfo?hcno=DB5 DF74A820991A754E40878CD3DA3B2), we extracted place/ location element entities and constructed an ontology hierarchy of location elements. The ontology structure of the emergency place/location element in the EOM-NPOSESs is shown in Figure 3.

The object element of an emergency is a collection of objects participating in the emergency and in the dissemination of public opinion regarding the emergency, such as public opinion platforms and key users during the spread of online public opinion. Public opinion platforms include mainstream news portals and social media platforms (Weibo and WeChat). Key users are mined and analyzed based on the results of previous key user mining methods [21].

The action element of an emergency refers to the trigger word of an emergency [12]. Trigger words for emergencies can be extracted using named entity recognition.

The element of emotional attitude of an emergency describes the emotional attitude of the netizens (positive, neutral, and negative) toward that emergency. This element was analyzed using sentiment analysis.

The level of an emergency is determined based on the impact of the emergency, level of harm, level of network public opinion, relevant laws, and regulations.

\subsubsection{OWL Description and Modeling of Emergency} Ontology. This section presents the OWL modeling of emergency ontology through the extended OWL. The relationship between emergencies was established using the ObjectProperty type. Each specific emergency can be associated with other emergencies through several ObjectProperties. ObjectProperty is used to restrict the relationship between emergencies. There are three types of restrictions: "all values from," "some values from," and "has value." The restriction "all values from" implies that the specified attribute can only be obtained from the specified class; "some values from" implies that the specified attribute is obtained from the specified part of the class; and "has value" implies that the specified attribute can only take a fixed value. For instance, the tsunami emergency defines an ObjectProperty attribute that represents the following relationship: its restriction is some values from an earthquake, which represent tsunamis that occur with an earthquake.

In this study, the time element and place/location element of an emergency were considered as the subattributes of ObjectProperty. Further, the object, action, level, and emotional elements of an emergency were regarded as the subattributes of DataProperty. The lower emergency set in the ontology was associated with the corresponding upper emergency set with OWL: equivalentClass.

\section{Experimental Evaluation}

In this section, we first summarize the important emergencies and emergency elements obtained from certain emergency websites, news portals, Weibo, and WeChat. Thereafter, we compare the EOM-NPOSESs with other emergency ontology models. Finally, we consider the COVID-19 emergency as an example for constructing an ontology model. Combined with the ontology model, we also explain the design of some rule reasoning strategies and propose a COVID-19 emergency application based on EOM-NPOSESs using a geographic information system (GIS) platform.

4.1. Data Acquisition. In our experiments, the Qingbo public opinion big data platform (https://yuqing.gsdata.cn/) was used to collect COVID-19 public health emergency data based on related keywords. Its main data sources include news portals, Weibo, and WeChat. Simultaneously, a crawler program was compiled to crawl the China Emergency Service Network (https://www.52safety.com/), the National Emergency Broadcasting Network (https://www. cneb.gov.cn/), and Zhiwei (https://xgml.zhiweidata.net).

\subsection{Experimental Results}

4.2.1. Comparative Analysis of Emergency Ontology Models. Previous studies have constructed ontology models and explored applications for emergencies. The comparison results of the related emergency ontology models are summarized in Table 1. "Model" represents the name of model, "Domain" represents the application field of the ontology model, "General" means that the model can be applied to all types of emergencies, and "Specific" means that the model can only be applied to a specific emergency. "Hierarchical relationship" and "Nonhierarchical relationship" denote whether or not the hierarchical and nonhierarchical emergency relationships are defined in the model. "Emergency knowledge" and "Public opinion knowledge", respectively, represent whether or not the emergency and public opinion management-related knowledge are included in the emergency ontology model. Traditional emergency ontology models use a single-dimensional concept to indicate emergencies, which makes it difficult to incorporate emergency and public opinion knowledge. The emergency ontology model proposed in this study includes emergency knowledge, public opinion knowledge, as well as hierarchical and nonhierarchical relationships between emergencies, which can be applied to the fields of emergency management and public opinion response.

\subsubsection{Emergency Ontology Model Analysis for COVID-19.} Coronavirus disease (COVID-19) refers to the type of pneumonia caused by the 2019 new coronavirus infection. In December 2019, some hospitals in Wuhan discovered 


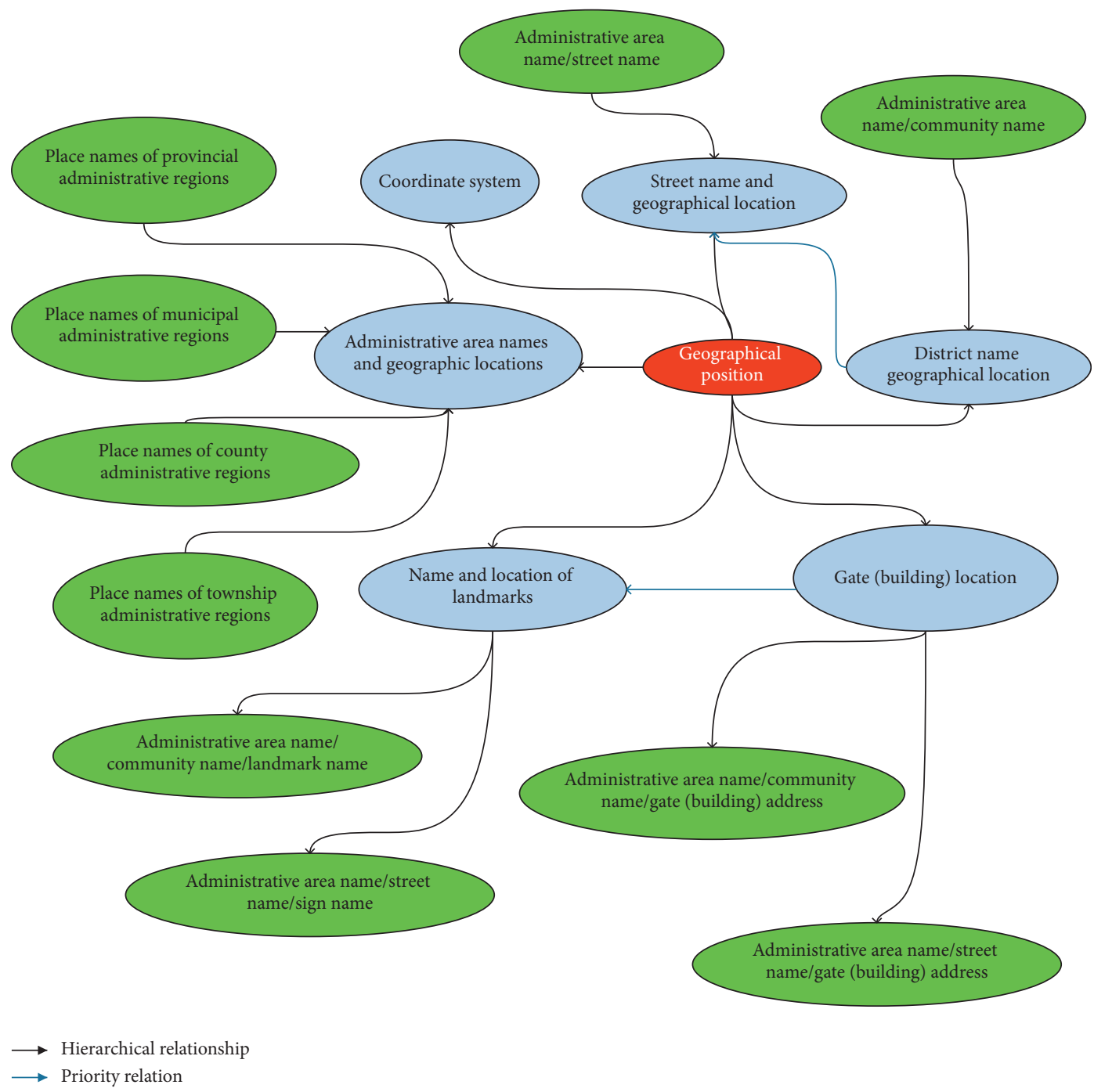

FIGURE 3: Ontology structure of emergency place/location element in EOM-NPOSESs.

multiple unexplained pneumonia cases with a history of exposure to the South China Seafood Market. COVID-19 has now been confirmed as an acute respiratory infectious disease caused by the 2019 new coronavirus infection. The Director-General of the World Health Organization Tedros Adhanom Ghebreyesus held a press conference in Geneva to explain that the pneumonia epidemic caused by the new coronavirus constituted a public health emergency of international concern. The National Health Commission Director stated that COVID-19 is a major public emergency with the fastest spread, the widest range of infections, and the most difficult prevention since its founding. We used the COVID-19 emergency as an example to model the relationship between COVID-19 emergencies and the emergency elements to verify the validity of the emergency ontology model proposed in this study. Figure 4 shows the nonhierarchical relationships of the emergency ontology model for COVID-19. For convenience, only some emergencies are presented in Figure 4. Figure 5 shows the emergency knowledge representation of the emergency ontology model for COVID-19. Each emergency has a description of six elements: time, place/location, object, action, emotional attitude, and level.

4.2.3. Emergency Ontology Reasoning for COVID-19. Ontology reasoning is based on rules. Common reasoning engines can use grammatical structures to realize ontology reasoning. Jena's internal rule engine provides forward, backward, and hybrid models. In addition, custom inference rules can be used to construct hierarchical relationships and attribute corresponding axioms in the class diagram, such as expressing the relationship between characters and events in emergencies. Both the premise and conclusion comprise one or more atomic rules.

Figure 6 shows some key user elements of the emergency ontology model for COVID-19. Emergencies A (Tokyo Olympics faces cancellation of attention) and B (IOC allowed the postponement of the Tokyo Olympics) have the following relationship: If the network public opinion 
TABLE 1: Comparison results of emergency ontology models.

\begin{tabular}{lccccc}
\hline Model & Domain & $\begin{array}{c}\text { Hierarchical } \\
\text { relationship }\end{array}$ & $\begin{array}{c}\text { Nonhierarchical } \\
\text { relationship }\end{array}$ & $\begin{array}{c}\text { Emergency } \\
\text { knowledge }\end{array}$ & $\begin{array}{c}\text { Public opinion } \\
\text { knowledge }\end{array}$ \\
\hline EOP [17] & Universal & $\mathrm{Y}$ & $\mathrm{Y}$ & $\mathrm{Y}$ & $\mathrm{Y}$ \\
Literature [18] & Universal & $\mathrm{Y}$ & $\mathrm{Y}$ & $\mathrm{N}$ & $\mathrm{N}$ \\
SEM [19] & Universal & $\mathrm{Y}$ & $\mathrm{N}$ & $\mathrm{N}$ & $\mathrm{N}$ \\
Literature [15] & Universal & $\mathrm{Y}$ & $\mathrm{Y}$ & $\mathrm{Y}$ & $\mathrm{N}$ \\
Empathi [20] & Specific & $\mathrm{Y}$ & $\mathrm{Y}$ & $\mathrm{Y}$ & $\mathrm{N}$ \\
EOM-NPOSESs & Universal & $\mathrm{Y}$ & $\mathrm{N}$ & $\mathrm{Y}$ & $\mathrm{N}$ \\
EmergencyFire [13] & Specific & $\mathrm{N}$ & & & $\mathrm{Y}$ \\
\hline
\end{tabular}

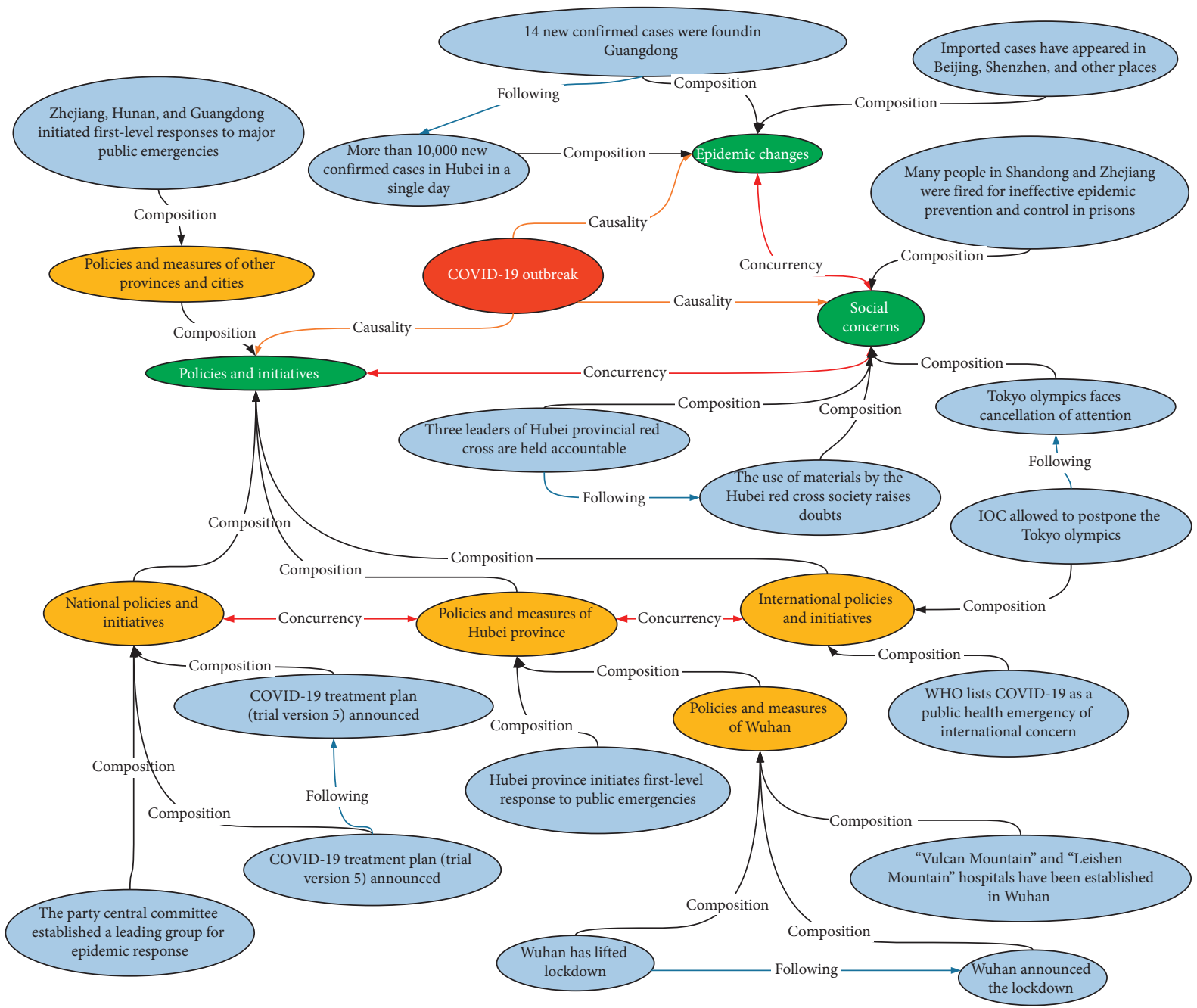

FIGURE 4: Nonhierarchical relationships of emergency ontology model for COVID-19.

dissemination platform and key users object element of Emergency B have been extracted, we can design key user inference rules for Emergency A. The key user inference rule is as follows.

[ruleHoldShare: (?) A: Following this relationship ?B) (? B: Key user ?c) $\longrightarrow($ ?A: Key user ?c)]

As shown in Figure 6, headline news is the key user of Emergency B. If key users of Emergency A are not found, we can infer that the key user of event $\mathrm{A}$ is headline news. The reasoning results of the key users are consistent with those of the EOM-NPOSESs.

In addition, we verified other reasoning rules based on the EOM-NPOSESs. As shown in Figure 4, Emergency $\mathrm{C}$ (policies and measures of Hubei province) has a composition relationship with Emergency D (policies and measures of Wuhan city) and Emergency $\mathrm{C}$ has a 


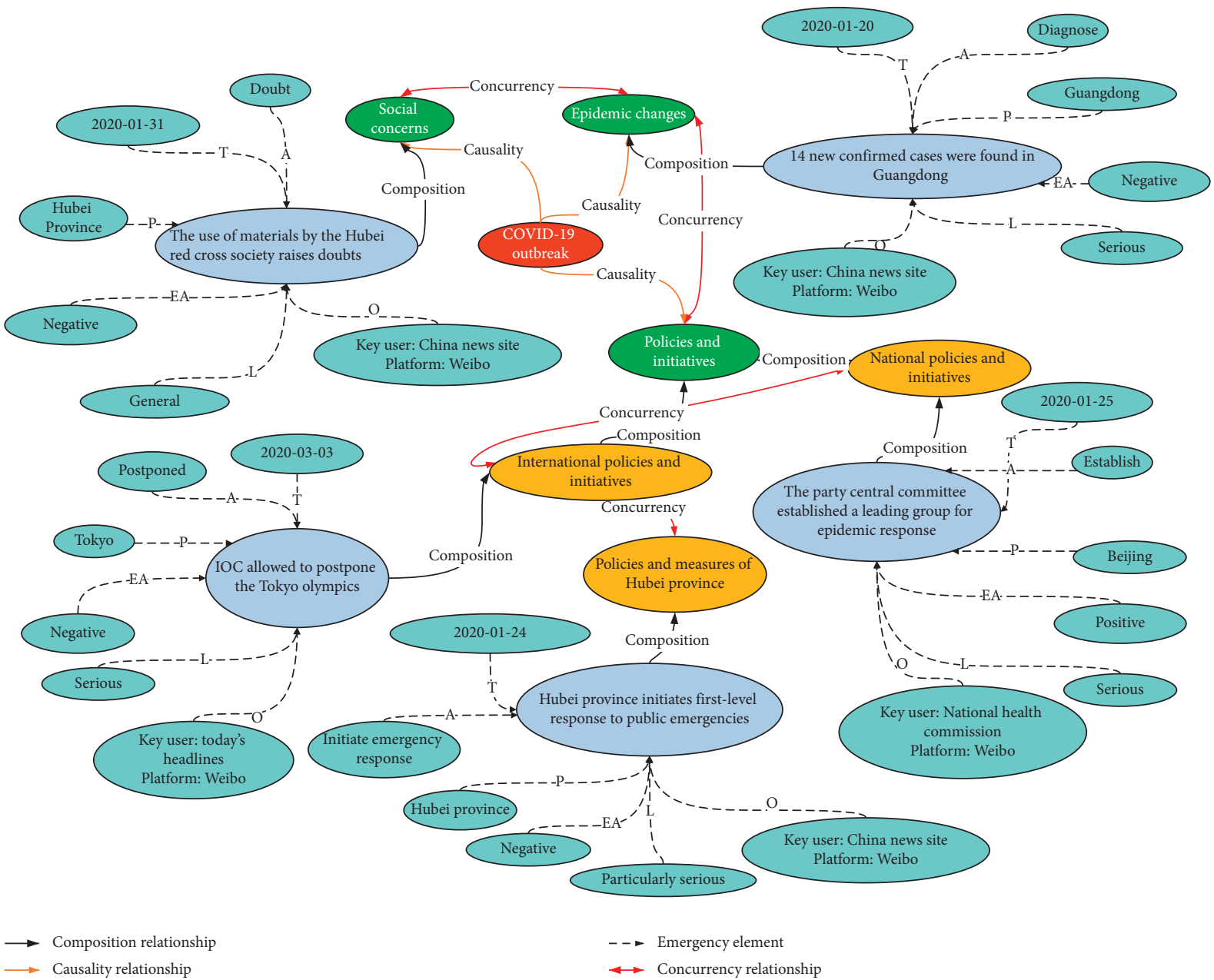

FIGURE 5: Emergency knowledge representation of emergency ontology model for COVID-19.

composition relationship with Emergency E (policies and measures of Huangshi city). Emergency D and the location element of Emergency $\mathrm{E}$ were extracted. As Wuhan and Huangshi cities are in Hubei province, we can refer to the policies and measures adopted in the Wuhan city to determine the epidemic policies and measures related to Emergency E. The customizable rules are as follows.

[ruleHoldShare: (?) a1: parallel relationship?a2) (?E: location element?a1) (?D: location element?a2)" + “(?c2: composition relationship,? D) $\longrightarrow$ (?E: refer to ?c2)]

The ontology model proposed in this study presents the relationship between time, object, action, and the entire process of evolution of public opinion-related emergencies. Owing to the uncertainty of online public opinion and the development of emergencies, the reasoning results obtained using this model can provide a reference for emergency public opinion response and control departments. In addition, reasoning based on this model can help formulate emergency policies and measures for different emergencies. In other words, the emergency policies and measures selected by this model are helpful for guiding the development of an emergency response.

We combined our EOM-NPOSESs with a GIS platform to realize an emergency response prototype system. As shown in Figure 7, the developed system can dynamically display COVID-19 emergencies according to the timeline. In this system, the dynamic knowledge of an emergency is automatically instantiated, and dynamic knowledge reasoning and rapid knowledge support for emergency response actions are provided. To verify the role of this prototype system, we take the example of the COVID-19 emergency policies and measures in a specific city. The demonstration application of emergency policies and measures reasoning is shown in Figure 8. The reasoning results were found to be consistent with the actual results, 


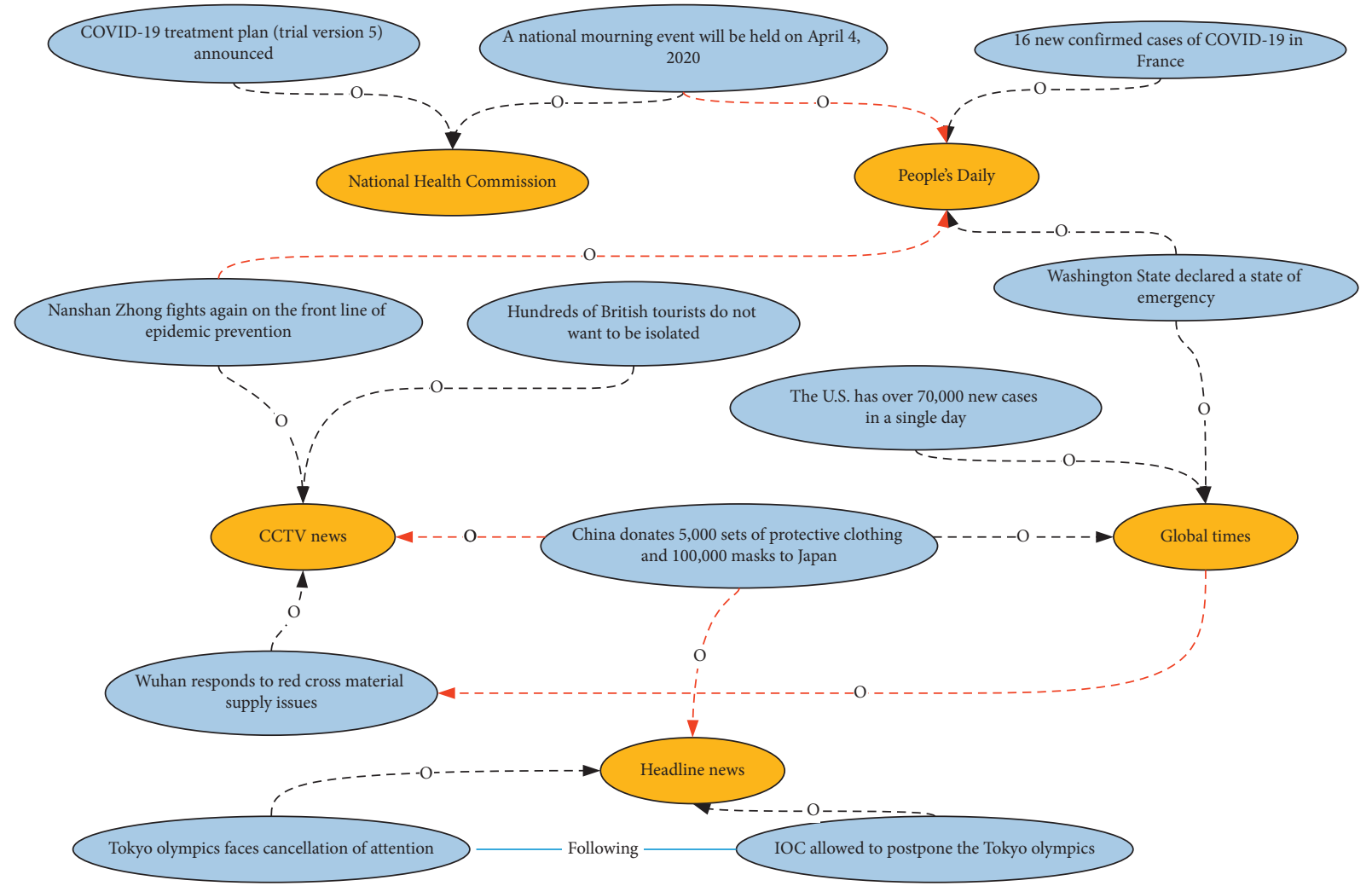

FIGURE 6: Some key user elements of emergency ontology model for COVID-19.

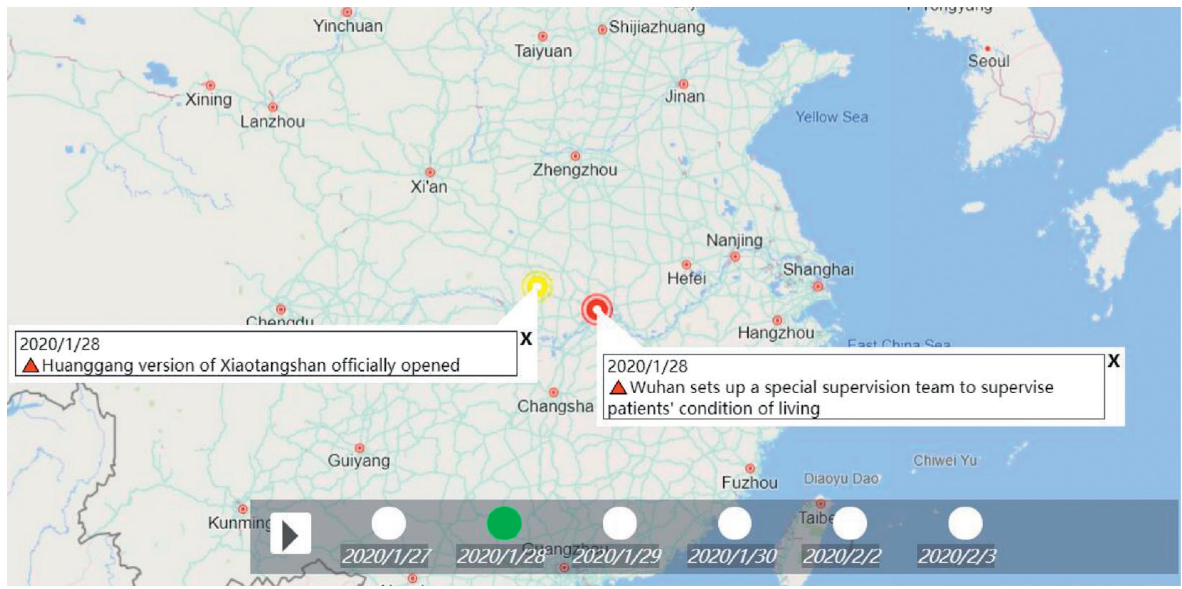

FIgURE 7: COVID-19 emergencies according to the timeline in the emergency response prototype system. 


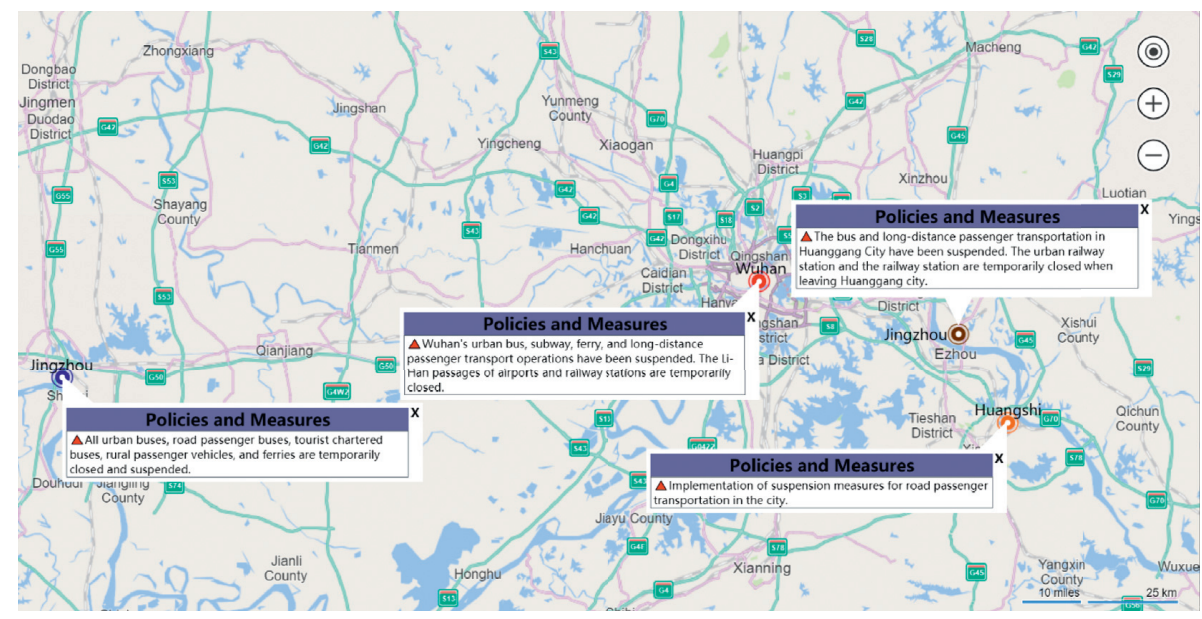

Figure 8: Demonstration application of emergency policies and measures reasoning.

and the usability of the EOM-NPOSESs model in practical applications was verified.

\section{Conclusions}

In this study, we constructed an emergency dataset and proposed EOM-NPOSESs. An emergency class and emergency class element construction method were proposed to construct the EOM-NPOSESs. Protégé was utilized to complete the OWL modeling of an emergency ontology through the extended OWL. Based on the constructed EOMNPOSESs, the reasoning rules of COVID-19 emergency were set. We also developed an emergency response prototype system to verify the availability of the EOM-NPOSESs. The experimental results demonstrate that the EOMNPOSESs can not only describe the semantic relationship between emergencies and elements of emergency but also perform semantic logical reasoning on different emergencies. In our future work, we will focus on extending our EOM-NPOSESs model and extracting the relations of emergency elements for multimedia data. We will also further investigate effective strategies for emergency relationship extraction.

\section{Data Availability}

The data used to support the findings of this study are available from the corresponding author upon request.

\section{Conflicts of Interest}

The authors declare that they have no conflicts of interest regarding the publishing of the present study.

\section{Acknowledgments}

This work was supported in part by the Key-Area Research and Development Program of Guangdong Province 2020B0101360001, and the Peng Cheng Laboratory Project (Grant no. PCL2021A02).

\section{References}

[1] C. You, D. Zhu, Y. Sun et al., "SNES: social-network-oriented public opinion monitoring platform based on ElasticSearch," Computers, Materials \& Continua, vol. 61, no. 3, pp. 12711283, 2019.

[2] W. Yang, S. Rehman, and W. Que, "Identifying event-specific opinion leaders by local weighted LeaderRank," Intelligent Automation \& Soft Computing, vol. 26, no. 6, pp. 1561-1574, 2020.

[3] G. Sun, S. Bin, M. Jiang et al., "Research on public opinion propagation model in social network based on blockchain," Computers, Materials \& Continua, vol. 60, no. 3, pp. 10151027, 2019.

[4] F. Ali, A. Ali, M. Imran et al., "Traffic accident detection and condition analysis based on social networking data," Accident Analysis \& Prevention, vol. 151, Article ID 105973, 2021.

[5] G. Dong, J. Gao, L. Huang, and C. Shi, "Online burst events detection oriented real-time microblog message stream," Computers, Materials \& Continua, vol. 60, no. 1, pp. 213-225, 2019.

[6] J. Su, Z. Sheng, A. X. Liu, Y. Han, and Y. Chen, "Captureaware identification of mobile RFID tags with unreliable channels," IEEE Transactions on Mobile Computing, pp. 1-141, 2020.

[7] S. H. Alsamhi, F. A. Almalki, O. Ma, M. S. Ansari, and M. C. Angelides, "Performance optimization of tethered balloon technology for public safety and emergency communications," Telecommunication Systems, vol. 75, no. 2, pp. 235-244, 2020.

[8] F. Ali, S. El-Sappagh, S. M. Riazul Islam et al., "An intelligent healthcare monitoring framework using wearable sensors and social networking data," Future Generation Computer Systems, vol. 114, pp. 23-43, 2019.

[9] S. Wang, Q. Peng, and H. Liang, "An event ontology model research for environmental pollution emergencies," International Journal of Ambient Computing and Intelligence, vol. 11, no. 4, pp. 38-56, 2020.

[10] M. Khan, P. Roy, I. Matin, M. Rabbani, and R. Chowdhury, "An adaptive governance and health system response for the COVID-19 emergency," World Development, vol. 137, Article ID 105213, 2021.

[11] H. Wang, H. Li, and H. Li, "Research of relation extraction method of civil aviation emergency domain ontology," 
Journal of Frontiers of Computer Science \& Technology, vol. 14, no. 2, pp. 285-293, 2020.

[12] F. Ali, S. El-Sappagh, and D. Kwak, "Fuzzy ontology and LSTM-based text mining: a transportation network monitoring system for assisting travel," Sensors, vol. 19, no. 2, Article ID 234, 2019.

[13] K. Bitencourt, F. Durão, and M. Mendonça, "EmergencyFire: an ontology for fire emergency situations," in Proceedings of the 21st Brazilian Symposium on Multimedia and the Web, pp. 73-76, Manaus, Brazil, October 2015.

[14] W.-j. Wang, P. Yang, and C.-x. Dong, "Study and application of emergency case ontology model," Journal of Computer Applications, vol. 29, no. 5, pp. 1437-1440, 2009.

[15] W. Zhu and Z. Liu, "Emergency domain knowledge modeling based on event ontology," Computer Engineering and Application, vol. 54, no. 21, pp. 148-155, 2018.

[16] Z.-j. Ni, L. Rong, N. Wang, and S. Cao, "Knowledge model for emergency response based on contingency planning system of China," International Journal of Information Management, vol. 46, pp. 10-22, 2019.

[17] W. Liu, Y. Tan, Y. Ding, Y. J. Zhang, and Z. T. Liu, "An ontology pattern for emergency event modeling," in Proceedings of the 2016 IEEE 14th Intl Conference on Dependable, Autonomic and Secure Computing, pp. 151-156, Auckland, New Zealand, August 2016.

[18] Y. Tan, W. Liu, Z. Yang, X. Du, and Z. Liu, "Pattern-based ontology modeling and reasoning for emergency system," IEICE Transactions on Information and Systems, vol. E101-D, no. 9, pp. 2323-2333, 2018.

[19] W. R. van Hage, V. Malaisé, R. Segers, L. Hollink, and G. Schreiber, "Design and use of the simple event model (SEM)," Journal of Web Semantics, vol. 9, no. 2, pp. 128-136, 2011.

[20] M. Gaur, S. Shekarpour, A. Gyrard, and A. Sheth, "Empathi: an ontology for emergency managing and planning about hazard crisis," in Proceedings of the 13th IEEE International Conference on Semantic Computing, pp. 396-403, Newport Beach, CA, USA, January 2018.

[21] G. Dong, W. Yang, F. Zhu, and W. Wang, "DPBT: a system for detecting pacemakers in burst topics," in Proceedings of the 17th International Conference on Web-Age Information Management, pp. 537-540, Nanchang, China, June 2016.

[22] G. Dong, W. Yang, F. Zhu, and W. Wang, "Discovering burst patterns of burst topic in twitter," Computers \& Electrical Engineering, vol. 58, no. 2, pp. 551-559, 2017.

[23] L. Liu, F. Mu, P. Li et al., "NeuralClassifier: an open-source neural hierarchical multi-label text classification toolkit," in Proceedings of the 57th Annual Meeting of the Association for Computational Linguistics, pp. 87-92, Florence, Italy, January 2019. 